\title{
Woody flora of Uruguay: inventory and implication within the Pampean region
}

\author{
Federico Haretche ${ }^{1}$, Patricia Mai ${ }^{1}$ and Alejandro Brazeiro ${ }^{1,2}$
}

Recebido em 9/02/2012. Aceito em 24/04/2012

\section{RESUMO}

(Flora lenhosa do Uruguai: inventário e implicação na região Pampeana). Contar com um conhecimento adequado da flora é fundamental para o desenvolvimento de investigação em diversos campos disciplinares. Neste contexto, nosso trabalho surge da necessidade de atualizar e melhorar a informação disponível sobre a flora lenhosa nativa do Uruguai. Nossos objetivos são determinar objetivamente a flora lenhosa uruguaia (arbustos e árvores), avaliar a completude do inventário e explorar sua similaridade com regiões vizinhas. Ao analisar a flora do Uruguai, produzimos definições operacionais de arbustos e árvores e obtivemos uma lista de 313 espécies (57 famílias, 124 gêneros). Usando 7.418 registros de distribuição, geramos curvas acumulativas de riqueza de espécies para estimar o potencial máximo de riqueza de espécies em escala nacional e local. Concluímos que a completude a nível nacional é elevado (89-95\%), mas em escala local é menor e bastante heterogêneo. Existem ainda grandes áreas sem dados ou com pouca informação. Encontramos que as espécies arbóreas do Uruguai, comparativamente, apresentam similaridade elevada com a Província de Entre Rios (Argentina), média com a Província de Buenos Aires (Argentina) e baixa com o Rio Grande do Sul (Brasil). Conclui-se que a riqueza de árvores e arbustos da flora uruguaia é maior do que a esperada para uma região de pradarias, e as diferenças encontradas nos indíces de similaridade com as floras lenhosas vizinhas estimulam estudos futuros para reavaliar o esquema fitogeográfico da região.

Palavras chave: Distribuição geográfica; Completude do inventário; Arbustos e árvores; Fitogeografia do Uruguai

\begin{abstract}
(Woody flora of Uruguay: inventory and implication within the Pampean region). Adequate knowledge of a flora is fundamental for furthering research in several disciplines. In this context, our work arises from the necessity to update and improve available information on the native woody flora of Uruguay. The goals of this study were to objectively determine the Uruguayan native woody flora (shrub and trees), to evaluate its inventory completeness, and to explore its similarity with neighboring regions. By analyzing the Uruguayan flora we produced working definitions of shrubs and trees, and obtained a list of 313 species (57 families, 125 genera). Using 7418 distribution records, we generated cumulative species richness curves to estimate maximum species richness at national and local scales. We conclude that the completeness at the national level is high (89-95\%), but at the local scale is lower and quite heterogeneous. There are still large areas without data or with little information. We found that comparatively, the similarity between Uruguayan tree species and the Province of Entre Ríos (Argentina) was high, while similarity with the Province of Buenos Aires (Argentina) was medium, and with Rio Grande do Sul (Brazil) was low. In conclusion, richness of tree and shrub species of the Uruguayan flora is greater than expected for a grassland region, and the differences found in the similarity index with the neighboring woody floras will stimulate future studies to reevaluate phytogeographic schemes of the region.
\end{abstract}

Key words: Geographic distribution; Inventory completeness; Shrub and tree flora; Uruguayan phytogeography

\footnotetext{
${ }^{1}$ Universidad de la República, Facultad de Ciencias, Grupo Biodiversidad y Ecología de la Conservación, Uruguay, Montevideo

${ }^{2}$ Author for correspondence: ecologiaap@gmail.com
} 


\section{Introduction}

Floristic studies and inventories attempt to document the biodiversity of a geographic region (Funk 2006). A well-produced flora constitutes a fundamental tool for research in many disciplines, such as ecology, biogeography, evolution and ethnobotany (Mori 1992; Funk 2006; Palmer et al. 1995), and provides a major source of information for conservation, land-use planning and sustainable use of ecosystems (Mori 1992; Palmer et al. 1995; Prance et al. 2000). "Taxonomic impediment" is the critical lack of taxonomic knowledge, and has been considered a major obstacle for conservation and sustainable use of biodiversity (Schnak \& López 2003; Crisci 2006; Funk 2006). In this context, our work arises from the necessity to update and improve the knowledge of native woody flora.

Enhanced knowledge of the woody flora of Uruguay will contribute substantially to a more thorough understanding of Uruguayan phytogeography. Uruguay has traditionally been considered a part of the Pampean Province, a region characterized by extensive grasslands or grass-dominated steppes (Cabrera \& Willink 1973; Morrone 2001). However, a recent study (Brussa \& Grela 2007) suggests that the number of tree and shrub species in the flora might be greater than that expected for the Pampean region. Trees and shrubs are characteristic elements of forests and shrublands, respectively, and represent $12 \%$ of all vascular plants in Uruguay, which add up to approximately 2750 species (Alonso \& Bassagoda 2002). In Uruguay, the surface occupied by native forests is approximately 820,000 ha (MGAP 2011), which represents almost $4 \%$ of the continental territory. The main types of forests present in the country are gallery forests, hill forests and open forests (Chebataroff 1960; Del Puerto 1987; Alonso \& Bassagoda 1999). Regarding shrublands, there is scarce information on the area occupied by such woody vegetation.

The study of Uruguayan woody species was initiated by European naturalists (e.g., P. Commerson, J. Tweedie, A. de Saint-Hilaire, F. Sellow) between the mid-XVIII and late-XIX centuries, when these naturalists made their first collections in Uruguay (Arechavaleta 1906; 1908a; 1908b; Del Puerto 1969). Later, local botanists started to contribute to the field, producing the first attempts towards a national flora [Larrañaga (1922; 1923; 1927), Gibert (1873), Arechavaleta (1898-1911), Herter (1930; 1943-1957), and Lombardo (1982-1984)]. There also have been studies that focus on particular plant groups in the form of monographs or floristic treatments of single families (e.g., Legrand 1936; 1943; 1968; Arrillaga et al. 1973; Izaguirre \& Beyhaut 1998; 2003; Marchesi 1983). Further, there have been contributions particularly focused on the woody flora of Uruguay (e.g., Lombardo 1946; 1964; Muñoz et al.1993; 2005; Alonso \& Bassagoda 2002; Brussa \& Grela 2007).

The main goals of this study were: (1) to determine the native woody flora (shrubs and trees) of Uruguay, (2) to evaluate its inventory completeness, and (3) to explore its floristic similarity with the neighboring regions.

\section{Material and methods}

We first reviewed the literature to search for parameters that could provide precise and objective definitions for shrub and tree, applicable for the proposed aims. This step was necessary due to the lack of clear criteria for elaborating a list of shrubs and trees species from the main bibliography of woody plants of Uruguay.

Definitions of trees are common in the literature. Font Quer (1957) defines a tree as a "woody plant, at least $5 \mathrm{~m}$ height, with a single stem named trunk (...)". According to Smith et al. (2004) a tree is "an erect, usually single-stemmed, woody plant $5 \mathrm{~cm}$ or more dbh (diameter at breast height); some trees may have multiple trunks but at least some of them are $5 \mathrm{~cm}$ or more in diameter." Sobral et al. (2006) refer to trees as "erect plants, with well defined and woody stem, with a diameter more than $5 \mathrm{~cm}$ measured at $1.30 \mathrm{~m}$ above the ground". Regarding shrubs, definitions are also relatively common in the literature. Font Quer (1957) defines a shrub as a "woody plant, less than $5 \mathrm{~m}$ height, without a main stem, because it is branched at the base". Smith et al. (2004) define a shrub as "a woody plant that is branched at the base or unbranched but less than $2 \mathrm{~m}$ tall'. In order to include tree-like plants such as tree ferns or some conspicuous monocotyledons, other authors (Ricker \& Hernández 2010) have broadened the definition of trees as: "trees and tree-like plants are defined broadly as perennial, self-supporting plants with an adult height of at least $5 \mathrm{~m}$ (without ascending leaves or inflorescences), and with one or several, erect stems with a diameter of at least $10 \mathrm{~cm}$ (measured at $1.3 \mathrm{~m}$ above the ground level)".

The definitions mentioned above do not completely fit the characteristics of species that comprise the woody flora of Uruguay, mainly because trees of the region tend to be short in height or have slim trunks. Therefore, we hereby propose pragmatic definitions for trees and shrubs, adapting the concepts of the definitions mentioned above, with the characteristics of the woody flora of Uruguay as follows: 1) tree and tree-like plants are terrestrial or hemiepiphyte plants, perennial, erect, with one or few well defined stems, partial or totally woody, of at least $5 \mathrm{~cm}$ in diameter measured at $1.3 \mathrm{~m}$ above the ground level and an adult height of at least $3 \mathrm{~m}$;2) shrubs are terrestrial or hemiepiphyte plants, perennial, erect or scandent, branched at the base or close to it, with a minimum height of $1 \mathrm{~m}$ above ground level and with aerial woody stems, persistent throughout the year.

These definitions attempt to be sufficiently broad to cover the greatest number of species that grow as trees in broad sense (trees and tree-like) and shrubs. Some species are impossible to classify definitively as trees or shrubs, due to variation in physiognomy or due to the influence of environmental conditions, particularly in this region (Alonso \& Bassagoda 2002). As such, these species are identified in this study as belonging to both life forms. 
To produce the list of taxa and generate a record database, we used the taxonomic category of species without considering infraspecific categories. Species were named according to Tropicos, botanical information system of the Missouri Botanical Garden - www.tropicos.org (2011) and to the list of Espécies da Flora do Brasil $2011 \mathrm{http}: / /$ floradobrasil.jbrj.gov.br/2011. The family classification follows APG III (2009). We used a total of 7418 herbarium specimens from MVFA (4504 specimens), MVJB (2145 specimens), MVM (732 specimens), and MVHC (23 specimens), whose determinations were made by Uruguayan specialists from the consulted herbaria. Specimens with doubtful identifications and/or uncertain localities were excluded. Herbarium acronyms follow Index Herbariorum (Holmgren \& Holmgren 1998). Location and other plant trait data were taken from specimen labels.

\section{Data analysis}

To analyze the geographic distribution of taxa included in this study, each record was geo-referenced, according to a grid system of 302 quadrants (approximately $22 \times 30 \mathrm{~km}$ ) at the scale 1:50.000 (SGM 1990). This information was integrated into a Geographic Information System (GIS).

The inventory completeness for tree and shrub species was assessed at the national level, using all the species included in our list. We first created species accumulation curves for the complete dataset of Uruguay. Then, we fitted the non-parametric model Chao 2 to estimate maximum species richness (with confidence interval of 95\%) using EstimateS 8.0 (Colwell 2006).

To assess the adequacy of sampling effort for a complete inventory of the woody flora at a local level (i.e., quadrant-scale) we estimated optimal sampling effort by analyzing the relationship between recorded richness and sampling effort (number of records). A moving average smoothing (lag=10) was fitted to this relationship, and the optimal sampling effort was considered the point where the curve achieved an asymptote. Beyond this point, the observed species richness does not increase consistently with sampling effort, suggesting the complete species assemblage has been registered. The estimation of optimal sampling effort was conducted using floristic regions based on woody plant distributions proposed by Grela (2004). The use of floristic regions takes into account the heterogeneity of flora and vegetation in the country, because areas with different diversity and/or woody vegetation surface may require different sampling effort for proper measurement. Grela (2004) identified two floristic regions with outstanding woody diversity: Western and Eastern Uruguay, the latter with two main subregions (referred here as "Eastern A" and "Eastern B"). The rest of the country (mainly central sectors) had lower woody species richness and was considered here the "Central region". The latter comprises 194 quadrants, while the Western covers 40 quadrants, the Eastern A, 22, and the Eastern B, 44. In addition, considering the optimal number of records by region, we calculated the percentage of quadrants sufficiently sampled in relation with the total quadrants of each region. This percentage involves the quadrants with a number of records same as or higher than the optimal number.

To evaluate tree richness similarity between Uruguay and neighboring regions, we used our definition of tree as the criteria to elaborate the species list and the species-region matrix. We analyzed the similarity, using the Jaccard Index, between different regions at species level, in particular for comparisons between Uruguay and the Entre Ríos and Buenos Aires Provinces in Argentina as well as the state of Rio Grande do Sul in Brazil. Information about tree species richness in these regions was obtained and adapted to the proposed criteria from Zuloaga et al. (2008) and Sobral et al. (2006). For all statistical analyses we used the software PAST 2.08 b (Hammer et al. 2001).

\section{Results}

\section{Woody flora of Uruguay}

The woody flora of Uruguay consists of 313 species, grouped in 57 families and 125 genera (Table 1). Families with the greatest number of species are Fabaceae (52), Asteraceae (42), Myrtaceae (26), Malvaceae (19), Solanaceae (13) and Euphorbiaceae (11). In regard to life form, the greatest number of tree species corresponds to Fabaceae (13), Myrtaceae (12), Salicaceae (7), Euphorbiaceae and Lauraceae (6); while for shrub species they include Asteraceae (41), Fabaceae (39), Malvaceae (18), Myrtaceae (14) and Solanaceae (12).

\section{Cumulative sampling effort distribution}

The accumulated richness curve shows an asymptote at the national scale approaching the maximum number of species $(\mathrm{N}=313)$. The Chao 2 curve also shows an asymptotic pattern, indicating convergence of the estimation procedure (Fig. 1). Estimated maximum richness (mean) based on the Chao 2 model was 328.9 species, almost 16 species more than observed richness (313), indicating the degree of completeness is $95.1 \%$. However, considering the upper bound of the confidence interval, maximum species richness could reach 351.9 species, and thus there could still be 39 unknown species, resulting in a degree of completeness of $88.9 \%$.

The recorded richness per quadrant plotted against number of records shows an asymptotic curve for each region (Fig. 2). In all cases in the range of low records per quadrant, recorded richness increases proportionally with sampling effort; however, beyond a certain value (variable by region) their relationship becomes independent. This suggests that the optimal sampling effort is approximately 86 records per quadrant in the Western region, 56 records per quadrant in the Central region, and 183 and 96 records 
Table 1. Shrub and tree species of Uruguay. The codes of life forms are as follow, s: shrub, t: tree, s/t: shrub or tree, s- fern: shrub fern, $t$ - fern: tree fern

\begin{tabular}{|c|c|c|}
\hline Family / Species & Life form & Reference specimen \\
\hline \multicolumn{3}{|l|}{ ACANTHACEAE } \\
\hline Justicia brasiliana Roth & s & Rosengurtt B-7212 (MVFA) \\
\hline J. tweediana (Nees) Griseb. & s & Del Puerto \& Marchesi s.n.(MVFA 5661) \\
\hline Poikilacanthus glandulosus (Nees) Ariza Esp. & s & Del Puerto \& Marchesi s.n.(MVFA 10378) \\
\hline Ruellia angustiflora (Nees) Lindau ex Rambo & s & Alonso s.n.(MVM 2153) \\
\hline \multicolumn{3}{|l|}{ ADOXACEAE } \\
\hline Sambucus australis Cham. \& Schltdl. & $s / t$ & Baycé s.n.(MVFA 21652) \\
\hline \multicolumn{3}{|l|}{ ANACARDIACEAE } \\
\hline Lithraea brasiliensis Marchand & $s / t$ & Rosengurtt B-3040 (MVFA) \\
\hline L. molleoides (Vell.) Engl. & $\mathrm{t}$ & Brussa \& Grela s.n.(MVJB 25282) \\
\hline Schinus engleri F.A.Barkley & s & Rosengurtt et al. PE-4321 1/2 (MVFA) \\
\hline S. ferox Hassl. & s & Brussa \& Grela s.n.(MVFA 29273) \\
\hline S. lentiscifolius Marchand & $s / t$ & Rosengurtt B-2562 (MVFA) \\
\hline S. longifolius (Lindl.) Speg. & $\mathrm{t}$ & Callero \& Domínguez 144 (MVJB 22714) \\
\hline S. molle L. & $\mathrm{t}$ & Rosengurtt B-4976 (MVFA) \\
\hline S. sinuatus (Griseb.) Engl. & s & Marchesi \& Vignale s.n.(MVFA 23565) \\
\hline \multicolumn{3}{|l|}{ ANNONACEAE } \\
\hline Annona emarginata (Schltdl.) H.Rainer & $s / t$ & Del Puerto s.n.(MVFA 3255) \\
\hline A. maritima (Záchia) H.Rainer & s & Brussa s.n.(MVJB 21987) \\
\hline \multicolumn{3}{|l|}{ APOCYNACEAE } \\
\hline Aspidosperma quebracho-blanco Mart. \& Zucc. & $\mathrm{t}$ & Marchesi \& Vignale s.n.(MVFA 26939) \\
\hline Tabernaemontana catharinensis A. DC. & $\mathrm{t}$ & Del Puerto s.n.(MVFA 12393) \\
\hline \multicolumn{3}{|l|}{ AQUIFOLIACEAE } \\
\hline Ilex dumosa Reissek & $\mathrm{t}$ & Marchesi et al. s.n.(MVFA 17520) \\
\hline I. paraguariensis A.St.-Hil. & $\mathrm{t}$ & Lombardo et al. s.n.(MVFA 9115) \\
\hline \multicolumn{3}{|l|}{ ARECACEAE } \\
\hline Butia capitata (Mart.) Becc. & $\mathrm{t}$ & Berro 2887(MVFA) \\
\hline B. paraguayensis (Barb.Rodr.) L.H.Bailey & s & Delfino et al. s.n.(MVJB 20453) \\
\hline B. yatay (Mart.) Becc. & $\mathrm{t}$ & Berro 4193 (MVFA) \\
\hline Butyagrus nabonnandii (Prosch.) Vorster & $\mathrm{t}$ & Lombardo 4868 (MVJB) \\
\hline Trithrinax campestris (Burmeist.) Drude \& Griseb. & $s / t$ & Berro 371 (MVFA) \\
\hline Syagrus romanzoffiana (Cham.) Glassman & $\mathrm{t}$ & Lombardo 2412 (MVJB) \\
\hline \multicolumn{3}{|l|}{ ASTERACEAE } \\
\hline Baccharis anomala DC. & s & Rosengurtt B-4983a (MVFA) \\
\hline B. arenaria Baker & s & Marchesi 1311 (MVFA) \\
\hline B. articulata (Lam.) Pers. & s & Rosengurtt et al. PE-5554 (MVFA) \\
\hline B. breviseta DC. & s & Rosengurtt B-3905 (MVFA) \\
\hline B. caprariifolia DC. & s & Bonifacino \& Systma 861 (MVFA) \\
\hline B. cultrata Baker & s & Bonifacino s.n.(MVFA 28868) \\
\hline B. dracunculifolia DC. & s & Legrand 755 (MVM) \\
\hline B. flabellata Hook. \& Arn. & s & Rosengurtt B-6005 (MVFA) \\
\hline B. gibertii Baker & s & Rosengurtt B-7782 (MVFA) \\
\hline B. longiattenuata A.S.Oliveira & $s / t$ & Brussa et al. s.n.(MVFA 32663) \\
\hline B. megapotamica Spreng. & $\mathrm{s}$ & Del Puerto \& Marchesi s.n.(MVFA 11075) \\
\hline
\end{tabular}


Table 1. Continuation.

\begin{tabular}{|c|c|c|}
\hline Family / Species & Life form & Reference specimen \\
\hline B. microcephala (Less.) DC. & s & Rosengurtt \& Gallinal 5846 (MVFA) \\
\hline B. microdonta DC. & s & Marchesi et al. s.n.(MVFA 28088) \\
\hline B. oxyodonta DC. & s & Izaguirre et al. s.n.(MVFA 28467) \\
\hline B. punctulata DC. & s & Rosengurtt et al. PE-5480 (MVFA) \\
\hline B. spicata (Lam.) Baill. & s & Baycé et al. s.n.(MVFA 24648) \\
\hline B. subopposita DC. & s & Legrand 4039 (MVM) \\
\hline B. tridentada Vahl & s & Rosengurtt B-2695 (MVFA) \\
\hline B. vulneraria Baker & s & Del Puerto s.n.(MVFA 12201) \\
\hline Calea clematidea Baker & s & Bonifacino s.n.(MVFA 25549) \\
\hline Carelia berroi Hutch. & s & Alonso 619 (MVM 2235) \\
\hline C. cistifolia Less. & s & Rosengurtt B-6670 (MVFA) \\
\hline Eupatorium buniifolium Hook. \& Arn. & s & Rosengurtt et al. PE-5659 (MVFA) \\
\hline E. cruciatum (Vell.) Ariza Esp. & s & Marchesi \& Vignale s.n.(MVFA 27251) \\
\hline E. ericoides DC. & s & Marchesi et al. s.n.(MVFA 27309) \\
\hline E. intermedium DC. & s & Nyffeler \& Eggli 1537 (MVJB 25058) \\
\hline E. laevigatum Lam. & s & Legrand 478 (MVM) \\
\hline E. polystachyum DC. & s & Rosengurtt B-4631 (MVFA) \\
\hline E. serratum Spreng. & s & Rosengurtt B-3090 (MVFA) \\
\hline E. subintegerrimum Malme & $s / t$ & Marchesi \& Grela s.n.(MVFA 27118) \\
\hline E. tremulum Hook. \& Arn. & s & Rosengurtt 10933 (MVFA) \\
\hline $\begin{array}{l}\text { Gochnatia polymorpha (Less.) Cabrera ssp. } \\
\text { ceanothifolia (Less.) Cabrera }\end{array}$ & $s / t$ & Rosengurtt B-2792 (MVFA) \\
\hline Heterothalamus alienus (L.) Kuntze & s & Rosengurtt B-1861 (MVFA) \\
\hline H. psiadioides Less. & s & Rosengurtt et al. 10019 (MVFA) \\
\hline Tessaria absinthioides (Hook. \& Arn.) DC. & s & Del Puerto \& Millot s.n.(MVFA 859) \\
\hline T. dodoneifolia (Hook. \& Arn.) Cabrera & s & Marchesi \& Vignale s.n.(MVFA 26670) \\
\hline T. integrifolia Ruiz \& Pav. & $\mathrm{t}$ & Brussa et al. s.n.(MVJB 22453) \\
\hline Trixis divaricata (Kunth) Spreng. & s & Del Puerto \& Marchesi s.n.(MVFA 5840) \\
\hline T. praestans (Vell.) Cabrera & s & Rosengurtt B-6668 (MVFA) \\
\hline Verbesina subcordata DC. & s & Brussa et al. s.n.(MVJB 22457) \\
\hline Vernonia nitidula Less. & s & Rosengurtt B-690 (MVFA) \\
\hline V. scorpioides (Lam.) Pers. & s & Del Puerto \& Marchesi s.n.(MVFA 5718) \\
\hline \multicolumn{3}{|l|}{ BERBERIDACEAE } \\
\hline Berberis laurina Billb. & s & Rosengurtt et al. PE-5047 (MVFA) \\
\hline B. ruscifolia Lam. & s & Brussa \& Grela s.n.(MVFA 29636) \\
\hline \multicolumn{3}{|l|}{ BIGNONIACEAE } \\
\hline Handroanthus heptaphyllus (Vell.) Mattos & $\mathrm{t}$ & Del Puerto \& Marchesi s.n.(MVFA 11443) \\
\hline \multicolumn{3}{|l|}{ BLECHNACEAE } \\
\hline Blechnum tabulare (Thunb.) Kuhn & $s$-fern & Brussa \& Muñoz s.n.(MVJB 22660) \\
\hline \multicolumn{3}{|l|}{ BORAGINACEAE } \\
\hline Cordia americana (L.) Gottschling \& J.S. Mill. & $\mathrm{t}$ & Caram s.n.(MVFA 18593) \\
\hline C. ecalyculata Vell. & $\mathrm{t}$ & Brussa \& Grela s.n.(MVJB 25070) \\
\hline Heliotropium transalpinum Vell. & s & Del Puerto s.n.(MVFA 2040) \\
\hline Tournefortia paniculata Cham. & s & Del Puerto \& Marchesi s.n.(MVFA 15891) \\
\hline
\end{tabular}


Table 1. Continuation.

\begin{tabular}{|c|c|c|}
\hline Family / Species & Life form & Reference specimen \\
\hline Varronia curassavica Jacq. & s & Callero s.n.(MVJB 26107) \\
\hline V. dichotoma Ruiz \& Pav. & s & Brescia et al. s.n.(MVFA 21288) \\
\hline \multicolumn{3}{|l|}{ CACTACEAE } \\
\hline Cereus aff. stenogonus K. Schum. & s & Marchesi (MVFA s/n) \\
\hline C. hildmannianus K. Schum. & $s / t$ & Legrand $3223(M V M)$ \\
\hline Opuntia arechavaletae Speg. & s & Muñoz s.n.(MVJB 23001) \\
\hline O. megapotamica Arechav. & s & Nyffeler \& Eggli 1418 (MVJB 22376) \\
\hline Pereskia nemorosa Rojas Acosta & s & Herter 82375 (MVFA) \\
\hline \multicolumn{3}{|l|}{ CANNABACEAE } \\
\hline Celtis iguanea (Jacq.) Sargent & s & Marchesi \& Vignale s.n.(MVFA 24933) \\
\hline C.ehrenbergiana (Klotzsch) Liebm. & $\mathrm{t}$ & Del Puerto s.n.(MVFA 2294) \\
\hline \multicolumn{3}{|l|}{ CARDIOPTERIDACEAE } \\
\hline Citronella gongonha (Mart.) R.A.Howard & $s / t$ & Rosengurtt B-6658 (MVFA) \\
\hline C. paniculata (Mart.) R.A.Howard & $\mathrm{s} / \mathrm{t}$ & Marchesi et al. s.n.(MVFA 17607) \\
\hline \multicolumn{3}{|l|}{ CARICACEAE } \\
\hline Vasconcellea quercifolia A.St.-Hil. & $\mathrm{t}$ & Brescia et al. s.n.(MVFA 19141) \\
\hline \multicolumn{3}{|l|}{ CELASTRACEAE } \\
\hline Maytenus cassiniformis Reiss. & s & Brescia \& Marchesi s.n.(MVFA 3749) \\
\hline M. dasyclados Mart. & $s / t$ & Legrand $1886(M V M)$ \\
\hline M. muelleri Schwacke & $s / t$ & Marchesi \& Armand-Ugón s.n.(MVFA 22337) \\
\hline M. vitis-idaea Griseb. & $s / t$ & Marchesi ఓ Bonifacino s.n.(MVFA 26131) \\
\hline Schaefferia argentinensis Speg. & $s / t$ & Marchesi s.n.(MVFA 16991) \\
\hline \multicolumn{3}{|l|}{ COMBRETACEAE } \\
\hline Combretum fruticosum (Loefl.) Stuntz & s & Rosengurtt B-1066 (MVFA) \\
\hline Terminalia australis Cambess. & $s / t$ & Rosengurtt B-4915 (MVFA) \\
\hline \multicolumn{3}{|l|}{ CYATHEACEAE } \\
\hline Cyathea atrovirens (Langds. \& Fisch.) Domin & $\mathrm{t}$-fern & Brussa \& Grela s.n.(MVFA 29485) \\
\hline \multicolumn{3}{|l|}{ DICKSONIACEAE } \\
\hline Dicksonia sellowiana Hook. & $\mathrm{t}$-fern & Brussa \& Grela s.n.(MVFA 29228) \\
\hline \multicolumn{3}{|l|}{ EBENACEAE } \\
\hline Diospyros inconstans Jacq. & $\mathrm{t}$ & Marchesi s.n.(MVFA 21864) \\
\hline \multicolumn{3}{|l|}{ EPHEDRACEAE } \\
\hline Ephedra tweediana Fisch. et C.A. Mey emend. J. H. Hunz. & s & Rosengurtt et al. PE-5182 (MVFA) \\
\hline \multicolumn{3}{|l|}{ ERICACEAE } \\
\hline Agarista chlorantha (Cham.) G. Don & s & Brussa \& Grela s.n.(MVFA 29633) \\
\hline A. eucalyptoides (Cham. \& Schltdl.) D.Don & $\mathrm{t}$ & Brescia et al. s.n.(MVFA 17237) \\
\hline \multicolumn{3}{|l|}{ ERYTHROXYLACEAE } \\
\hline Erythroxylum microphyllum A.St.-Hil. & s & Brescia \& Marchesi s.n.(MVFA 3816) \\
\hline E. myrsinites Mart. & s & Brussa \& Escudero s.n.(MVJB 24464) \\
\hline \multicolumn{3}{|l|}{ ESCALLONIACEAE } \\
\hline Escallonia bifida Link \& Otto & $s / t$ & Baycé et al. s.n.(MVFA 23526) \\
\hline E. megapotamica Spreng. var. spiraeifolia (Cham. \& Schltdl.) Sleumer & s & Marchesi 1304 (MVFA) \\
\hline \multicolumn{3}{|l|}{ EUPHORBIACEAE } \\
\hline Actinostemon concolor (Spreng.) Müll.Arg. & $s / t$ & Brescia et al. s.n.(MVFA 16299) \\
\hline
\end{tabular}


Table 1. Continuation.

\begin{tabular}{|c|c|c|}
\hline Family / Species & Life form & Reference specimen \\
\hline Croton hilarii Baill. & s & Rosengurtt B-3149 (MVFA) \\
\hline C. lachnostephanus Baill. & s & Rosengurtt B-2690 (MVFA) \\
\hline C. urucurana Baill. & $\mathrm{t}$ & Del Puerto \& Borsani s.n.(MVFA 2420) \\
\hline C. uruguayensis Baill. & s & Rosengurtt et al. PE-5214 (MVFA) \\
\hline Manihot grahamii Hook. & $\mathrm{t}$ & Rosengurtt B-4975 (MVFA) \\
\hline Sapium glandulosum (L.) Morong & $\mathrm{t}$ & Rosengurtt 9918 (MVFA) \\
\hline S. haematospermum Müll.Arg. & $\mathrm{t}$ & Bonifacino et al. 1005 (MVFA) \\
\hline Sebastiania brasiliensis Spreng. & $\mathrm{t}$ & Brescia et al. s.n.(MVFA 16365) \\
\hline S. commersoniana (Baill.) L.B.Smith \& Downs & $\mathrm{t}$ & Osten 3828 (MVM) \\
\hline S. schottiana (Müll.Arg.) Müll.Arg. & s & Rosengurtt et al. PE-5022 (MVFA) \\
\hline \multicolumn{3}{|l|}{ FABACEAE } \\
\hline Vachellia astringens (Gillies ex Hook. \& Arn.) Speg. & $s / t$ & Del Puerto et al. s.n.(MVFA 9378) \\
\hline V. caven (Molina) Seigler \& Ebinger & s & Marchesi 1076 (MVFA) \\
\hline Senegalia bonariensis (Gillies ex Hook. \& Arn.) Seigler \& Ebinger & $\mathrm{s} / \mathrm{t}$ & Osorio s.n.(MVM 13395) \\
\hline S. praecox (Griseb.) Seigler \& Ebinger & $\mathrm{s} / \mathrm{t}$ & Grela \& Escudero s.n.(MVFA 28235) \\
\hline Aeschynomene montevidensis Vog. & s & Izaguirre \& Beyhaut s.n.(MVFA 19651) \\
\hline Albizia inundata (Mart.) Barneby \& J.W. Grimes & $\mathrm{t}$ & Del Puerto \& Borsani s.n.(MVFA 2447) \\
\hline Bauhinia forficata Link ssp. pruinosa (Vog.) Fortunato \& Wunderlin & $\mathrm{t}$ & Brussa et al. s.n.(MVJB 20728) \\
\hline Caesalpinia gilliesii (Wall. ex Hook.) D.Dietr. & s & Marchesi \& Armand-Ugón s.n.(MVFA 20950) \\
\hline Calliandra selloi (Spreng.) J.F. Macbr. & s & Brussa et al. s.n.(MVJB 22340) \\
\hline C. parvifolia (Hook. \& Arn.) Speg. & s & Marchesi s.n.(MVFA 21577) \\
\hline C. tweedii Benth. & s & Rosengurtt B-4840 (MVFA) \\
\hline Collaea stenophylla (Hook. \& Arn.) Benth. & s & Rosengurtt B-3281 (MVFA) \\
\hline Crotalaria micans Link & $\mathrm{s}$ & Arrillaga et al. 1948 (MVFA) \\
\hline Dalbergia frutescens (Vell.) Britton & s & Brussa et al. s.n.(MVJB 20519) \\
\hline Enterolobium contortisiliquum (Vell.) Morong & $\mathrm{t}$ & Del Puerto \& Marchesi s.n.(MVFA 5646) \\
\hline Erythrina crista-galli L. & $\mathrm{t}$ & Berro 359 (MVFA) \\
\hline Geoffroea decorticans (Gillies ex Hook. \& Arn.) Burkart & $\mathrm{s} / \mathrm{t}$ & Marchesi \& Armand-Ugón s.n.(MVFA 20371) \\
\hline Gleditsia amorphoides (Griseb.) Taub. & $\mathrm{t}$ & Haretche 118 (MVJB 26348) \\
\hline Indigofera suffruticosa Mill. & s & Marchesi et al. s.n.(MVFA 21919) \\
\hline Inga uraguensis Hook. \& Arn. & $\mathrm{t}$ & González s.n.(MVJB 22420) \\
\hline Lonchocarpus muehlbergianus Hassl. & $\mathrm{t}$ & Brussa et al. s.n.(MVJB 20641) \\
\hline L. nitidus (Vog.) Benth. & $\mathrm{t}$ & Rosengurtt et al. 10629 (MVFA) \\
\hline Mimosa adpressa Hook. \& Arn. & s & Rosengurtt et al. PE-5210 (MVFA) \\
\hline M. amphigena Burkart & s & Rosengurtt \& Gallinal 5816 (MVFA) \\
\hline M. australis Izaguirre \& Beyhaut & s & Rosengurtt B-2427 (MVFA) \\
\hline M. berroi Burkart & s & Izaguirre et al. s.n.(MVFA 19479) \\
\hline M. bifurca Benth. & s & Brescia \& Marchesi s.n.(MVFA3799) \\
\hline M. bimucronata (DC.) Kuntze & $\mathrm{s} / \mathrm{t}$ & Brussa \& Grela s.n.(MVFA 29869) \\
\hline M. bonplandii (Gillies ex Hook. \& Arn.) Benth. & s & Brussa et al. s.n.(MVJB 22456) \\
\hline M. burkartii Marchesi & s & Marchesi 1423 (MVFA) \\
\hline M. cruenta Benth. & s & Ren s.n.(MVFA 28449) \\
\hline M. daleoides Benth. & $\mathrm{s}$ & Izguirre \& Beyhaut s.n.(MVFA 28459) \\
\hline M. incana (Spreng.) Benth. & s & Marchesi \& Grela s.n.(MVFA 27141) \\
\hline
\end{tabular}


Table 1. Continuation

\begin{tabular}{|c|c|c|}
\hline Family / Species & Life form & Reference specimen \\
\hline M. magentea Izaguirre \& Beyhaut & s & Izguirre \& Beyhaut s.n.(MVFA 28454) \\
\hline M. ostenii Speg. ex Burkart & $s / t$ & Brussa et al. s.n.(MVJB 27403) \\
\hline M. pigra L. & s & Brussa \& Delfino s.n.(MVJB 22458) \\
\hline M. pilulifera Benth. & s & Berro 5734 (MVFA) \\
\hline M. ramulosa Benth. & s & Izguirre \& Beyhaut s.n.(MVFA 28453) \\
\hline M. sprengelii DC. & s & Rosengurtt B-5708 (MVFA) \\
\hline M. tweedieana Barneby ex Glazier \& Mackinder & s & Lema \& Marchesi s.n.(MVFA 8233) \\
\hline M. uraguensis Hook. \& Arn. & s & Rosengurtt B-946 (MVFA) \\
\hline Parapiptadenia rigida (Benth.) Brenan & $\mathrm{t}$ & Brussa \& Grela s.n.(MVFA 29163) \\
\hline Parkinsonia aculeata $\mathrm{L}$. & $s / t$ & Brussa \& Scarlato s.n.(MVJB 23110) \\
\hline Peltophorum dubium (Spreng.)Taub. & $\mathrm{t}$ & Lombardo 3040 (MVJB 9944) \\
\hline Poecilanthe parviflora Benth. & $\mathrm{t}$ & Arrillaga et al. 2016 (MVFA) \\
\hline $\begin{array}{l}\text { Pomaria rubicunda (Vogel) B.B. Simpson \& G.P. Lewis var. } \\
\text { rubicunda }\end{array}$ & s & Rosengurtt B-972 (MVFA) \\
\hline Prosopis affinis Spreng. & $\mathrm{t}$ & Del Puerto \& Marchesi s.n.(MVFA 5694) \\
\hline P. nigra (Griseb.)Hieron. & $\mathrm{t}$ & Marchesi s.n.(MVFA 20674) \\
\hline Senna corymbosa (Lam.) Irwin \& Barneby & $s / t$ & Rosengurtt B-85 (MVFA) \\
\hline $\begin{array}{l}\text { S. pendula (Willd.) H.S.Irwin \& Barneby var. paludicola H.S.Irwin } \\
\text { \& Barneby }\end{array}$ & $\mathrm{s} / \mathrm{t}$ & Del Puerto \& Borsani s.n.(MVFA 2428) \\
\hline Sesbania punicea (Cav.) Benth. & s & Berro 3824 (MVFA) \\
\hline S. virgata (Cav.) Pers. & s & Berro 3787 (MVFA) \\
\hline \multicolumn{3}{|l|}{ LAMIACEAE } \\
\hline Aegiphila brachiata Vell. & $s / t$ & Rosengurtt et al. 9989 (MVFA) \\
\hline Cunila incana Benth. & s & Izaguirre et al. s.n.(MVFA 21436) \\
\hline Vitex megapotamica (Spreng.) Mold. & $\mathrm{t}$ & Rosengurtt et al. 10020 (MVFA) \\
\hline \multicolumn{3}{|l|}{ LAURACEAE } \\
\hline Cinnamomum amoenum (Nees) Kosterm. & $\mathrm{t}$ & Rosengurtt B-8051 (MVFA) \\
\hline Nectandra angustifolia (Schrad.) Nees \& Mart.ex Nees & $\mathrm{t}$ & Brussa \& Lafarge s.n.(MVJB 27243) \\
\hline N. megapotamica (Spreng.) Mez & $\mathrm{t}$ & Del Puerto \& Marchesi s.n.(MVFA 3607) \\
\hline Ocotea acutifolia (Nees) Mez & $\mathrm{t}$ & Grela et al. s.n.(MVFA 27556) \\
\hline O. puberula (Rich.) Nees & $\mathrm{t}$ & Brescia \& Marchesi s.n.(MVFA 16975) \\
\hline O. pulchella Mart. & $\mathrm{t}$ & Grela et al. s.n.(MVFA 26251) \\
\hline \multicolumn{3}{|l|}{ LOGANIACEAE } \\
\hline Strychnos brasiliensis (Spreng.) Mart. & $\mathrm{t}$ & Brussa et al. s.n.(MVJB 21530) \\
\hline \multicolumn{3}{|l|}{ LORANTHACEAE } \\
\hline Tripodanthus acutifolius (Ruiz \& Pav.) Tiegh. & s & Rosengurtt B-2912 (MVFA) \\
\hline \multicolumn{3}{|l|}{ LYTHRACEAE } \\
\hline Heimia apetala (Spreng.) S.A. Graham \& Gandhi & s & Rosengurtt B-2888 (MVFA) \\
\hline H. salicifolia Link & s & Rosengurtt \& Gallinal 5832 (MVFA) \\
\hline \multicolumn{3}{|l|}{ MALPIGHIACEAE } \\
\hline Heteropterys glabra Hook. \& Arn. & s & Lombardo 4105 (MVJB 11540) \\
\hline \multicolumn{3}{|l|}{ MALVACEAE } \\
\hline Abutilon affine (Spreng.) G.Don & s & Del Puerto s.n.(MVFA 2393) \\
\hline A. grandifolium (Willd.) Sweet & s & Rosengurtt B-8008 (MVFA) \\
\hline A. pauciflorum A.St.-Hil. & s & Speroni \& Grela s.n.(MVFA 28074) \\
\hline
\end{tabular}


Table 1. Continuation.

\begin{tabular}{|c|c|c|}
\hline Family / Species & Life form & Reference specimen \\
\hline A. pictum (Gillies ex Hook. \& Arn.) Walp. & s & Berro 2825 (MVFA) \\
\hline A. umbelliflorum A.St.-Hil. & s & Brussa \& Grela s.n.(MVJB 23756) \\
\hline Byttneria urticifolia K.Schum. & s & Rosengurtt A-1336 (MVFA) \\
\hline Calyculogygas uruguayensis Krapov. & s & Del Puerto et al. s.n.(MVFA 9649) \\
\hline Hibiscus striatus Cav. & s & Del Puerto \& Millot s.n.(MVFA 1078) \\
\hline Luehea divaricata Mart. & $\mathrm{t}$ & Rosengurtt 8003 (MVFA) \\
\hline Pavonia betonicifolia C.Presl & s & Rosengurtt B-2503 (MVFA) \\
\hline P. distinguenda A.St.-Hil. \& Naudin & s & Legrand 1977 (MVM) \\
\hline P. glutinosa Krapov. \& Cristobal & s & Rosengurtt B-8138 (MVFA) \\
\hline P. hastata Cav. & s & Rosengurtt B-2877 (MVFA) \\
\hline P. orientalis Krapov. & s & Rosengurtt B-5309 (MVFA) \\
\hline P. rosengurtii Krapov. \& Cristobal & s & Berro 6071 (MVFA) \\
\hline P. sepium A.St.-Hil. & s & Rosengurtt \& Gallinal 5856 (MVFA) \\
\hline P. vitifolia Hochr. ex Chodat \& Hassler & s & Brussa et al. s.n.(MVJB 22528) \\
\hline P. xanthogloea Ekman & s & Rosengurtt et al. 10046 (MVFA) \\
\hline Sphaeralcea bonariensis (Cav.) Griseb. & s & Del Puerto \& Marchesi s.n.(MVFA 9011) \\
\hline \multicolumn{3}{|l|}{ MELASTOMATACEAE } \\
\hline Leandra australis (Cham.) Cogn.var. phaeotricha (Naudin) Cogn. & s & Brescia et al. s.n.(MVFA 19039) \\
\hline Miconia hyemalis A.St.-Hil. \& Naudin & $s / t$ & Grela \& Brussa 1108 (MVJB 21800) \\
\hline \multicolumn{3}{|l|}{ MELIACEAE } \\
\hline Guarea macrophylla Vahl ssp. spiciflora (A.Juss.) T.D.Penn. & $\mathrm{t}$ & Brussa et al. s.n.(MVJB 20487) \\
\hline Trichilia elegans A.Juss. & $\mathrm{t}$ & Brussa et al. s.n.(MVJB 21521) \\
\hline \multicolumn{3}{|l|}{ MORACEAE } \\
\hline Ficus cestrifolia Schott & $\mathrm{t}$ & Brussa \& Alvarez s.n.(MVJB 26092) \\
\hline F. luschnathiana (Miq.) Miq. & $\mathrm{t}$ & Brescia \& Marchesi s.n.(MVFA 3752) \\
\hline \multicolumn{3}{|l|}{ MYRTACEAE } \\
\hline Acca sellowiana (O.Berg) Burret & $s / t$ & Bonifacino \& Systma 845 (MVFA) \\
\hline Blepharocalyx salicifolius (Kunth) O.Berg & $s / t$ & Rosengurtt B-1542 (MVFA) \\
\hline Calyptranthes concinna DC. & $s / t$ & Brussa \& Grela s.n.(MVFA 29230) \\
\hline Campomanesia aurea O.Berg var. aurea & s & Marchesi et al. s.n.(MVFA 17589) \\
\hline C. xanthocarpa (Mart.) O.Berg & $\mathrm{t}$ & Brussa et al. s.n.(MVFA 29835) \\
\hline Eugenia involucrata DC. & $\mathrm{t}$ & Brussa et al. s.n.(MVJB 21557) \\
\hline E. mansoi O.Berg & $\mathrm{t}$ & Haretche 41 (MVJB 23997) \\
\hline E. pyriformis Cambess. var. uvalha (Cambess.) D.Legrand & $\mathrm{t}$ & Berro 3441 (MVFA) \\
\hline E. repanda O.Berg & $\mathrm{t}$ & Rosengurtt et al. 10585 (MVFA) \\
\hline E. uniflora $\mathrm{L}$. & $s / t$ & Brussa \& Grela s.n.(MVJB 24580) \\
\hline E. uruguayensis Cambess. & $\mathrm{t}$ & Rosengurtt \& Del Puerto 10660 (MVFA) \\
\hline Gomidesia palustris (DC.) Kausel & $\mathrm{t}$ & Marchesi et al. s.n.(MVFA 17637) \\
\hline Hexachlamys edulis (O.Berg) D.Legrand \& Kausel & $\mathrm{t}$ & Grela \& Escudero s.n.(MVFA 28233) \\
\hline Myrceugenia euosma (O.Berg) D.Legrand & s & Rosengurtt B-4801 (MVFA) \\
\hline M. glaucescens (Cambess.) D.Legrand \& Kausel & $s / t$ & Rosengurtt B-799 (MVFA) \\
\hline M. myrtoides O.Berg & s & Baycé et al. s.n.(MVFA 22367) \\
\hline Myrcia selloi (Spreng.) N. Silveira & $s / t$ & Rosengurtt B-990 (MVFA) \\
\hline M. verticillaris O.Berg & s & Brescia et al. s.n.(MVFA 17337) \\
\hline
\end{tabular}


Table 1. Continuation

\begin{tabular}{|c|c|c|}
\hline Family / Species & Life form & Reference specimen \\
\hline Myrcianthes cisplatensis (Cambess.) O.Berg & $\mathrm{t}$ & Rosengurtt et al. PE-5607 (MVFA) \\
\hline M. gigantea (D.Legrand) D.Legrand & $\mathrm{t}$ & Legrand 690 (MVFA) \\
\hline M. pungens (O.Berg) D.Legrand & $\mathrm{t}$ & Del Puerto s.n.(MVFA 2385) \\
\hline Myrciaria tenella (DC.) O.Berg & $s / t$ & Brussa \& Grela s.n.(MVFA 29334) \\
\hline Myrrhinium atropurpureum Schott var. octandrum Bentham & $s / t$ & Rosengurtt et al. PE-5024 (MVFA) \\
\hline Paramyrciaria delicatula (DC.) Kausel & s & Bonifacino \& Sancho 1045 (MVFA) \\
\hline Plinia rivularis (Cambess.) Rotman & $\mathrm{t}$ & Del Puerto \& Marchesi s.n.(MVFA 5926) \\
\hline Psidium cattleianum Sabine & $s / t$ & Del Puerto \& Marchesi s.n.(MVFA 6127) \\
\hline \multicolumn{3}{|l|}{ PHYLLANTHACEAE } \\
\hline Phyllanthus sellowianus Müll.Arg. & s & Osten 4269 (MVM) \\
\hline \multicolumn{3}{|l|}{ PHYTOLACCACEAE } \\
\hline Phytolacca dioica $\mathrm{L}$. & $\mathrm{t}$ & Baycé et al. s.n.(MVFA 26472) \\
\hline \multicolumn{3}{|l|}{ PICRAMNIACEAE } \\
\hline Picramnia sellowii Planch. & $\mathrm{t}$ & Brussa s.n.(MVJB 22867) \\
\hline \multicolumn{3}{|l|}{ POACEAE } \\
\hline Guadua chacoensis (Rojas) Londoño \& P.Peterson & s & Brussa et al. s.n.(MVJB 21047) \\
\hline G. trinii (Nees) Nees ex Rupr. & s & Del Puerto \& Marchesi s.n.(MVFA 5630) \\
\hline \multicolumn{3}{|l|}{ POLYGONACEAE } \\
\hline Coccoloba argentinensis Speg. & $s / t$ & Marchesi s.n.(MVFA 16967) \\
\hline Ruprechtia laxiflora Meisn. & $\mathrm{t}$ & Marchesi \& Armand-Ugón s.n.(MVFA 20493) \\
\hline R. salicifolia (Cham. \& Schltdl.) C.A.Mey. & $\mathrm{t}$ & Haretche 146 (MVJB 27067) \\
\hline \multicolumn{3}{|l|}{ PRIMULACEAE } \\
\hline Myrsine coriacea (Sw.) R.Br. ex Roem. \& Schult. & $\mathrm{t}$ & Rosengurtt A-1344 (MVFA) \\
\hline M. laetevirens (Mez) Arechav. & $\mathrm{t}$ & Marchesi et al. s.n.(MVFA 28049) \\
\hline M. parvifolia A.DC. & s & Del Puerto \& Marchesi s.n.(MVFA 5284) \\
\hline M. parvula (Mez) Otegui & $\mathrm{t}$ & Brussa \& Nicoli s.n.(MVJB 22428) \\
\hline M. umbellata Mart. & $\mathrm{t}$ & Brescia \& Marchesi s.n.(MVFA 4009) \\
\hline M. venosa A.DC. & $s / t$ & Marchesi \& Armand-Ugón s.n.(MVFA 21124) \\
\hline \multicolumn{3}{|l|}{ QUILLAJACEAE } \\
\hline Quillaja brasiliensis (A.St.-Hil. \& Tulasne) Mart. & $\mathrm{t}$ & Rosengurtt B-4963 (MVFA) \\
\hline \multicolumn{3}{|l|}{ RHAMNACEAE } \\
\hline Colletia paradoxa (Spreng.) Escal. & s & Rosengurtt et al. PE-5556 (MVFA) \\
\hline C. spinosissima Gmel. & s & Bonifacino s.n.(MVFA 25006) \\
\hline Condalia buxifolia Reissek & $\mathrm{s} / \mathrm{t}$ & Marchesi et al. s.n.(MVFA 28101) \\
\hline Discaria americana Gillies ex Hook. \& Arn. & s & Ziliani s.n.(MVFA 9462) \\
\hline Scutia buxifolia Reissek & $\mathrm{t}$ & Rosengurtt « Gallinal 5757 (MVFA) \\
\hline \multicolumn{3}{|l|}{ ROSACEAE } \\
\hline Prunus subcoriacea (Chod. \& Hassl.) Koehne & $\mathrm{s} / \mathrm{t}$ & Rosengurtt B-6681 (MVFA) \\
\hline \multicolumn{3}{|l|}{ RUBIACEAE } \\
\hline Cephalanthus glabratus (Spreng.) K.Schum. & s & Speroni s.n.(MVFA 24347) \\
\hline Chiococca alba (L.) Hitchc. & s & Brescia \& Marchesi s.n.(MVFA 4001) \\
\hline Guettarda uruguensis Cham. \& Schltdl. & $\mathrm{s} / \mathrm{t}$ & Grela et al. s.n.(MVFA 27612) \\
\hline Psychotria carthagenensis Jacq. & s & Legrand 2020 (MVM) \\
\hline Randia sp. ${ }^{3}$ & $\mathrm{~s} / \mathrm{t}$ & Del Puerto \& Marchesi s.n.(MVFA 5954) \\
\hline
\end{tabular}

${ }^{3}$ Randia sp. This species has been traditionally considered as Randia armata (Sw.) DC., but currently is considered a doubtful classification (Marchesi pers. com.). 
Table 1. Continuation.

\begin{tabular}{|c|c|c|}
\hline Family / Species & Life form & Reference specimen \\
\hline \multicolumn{3}{|l|}{ RUTACEAE } \\
\hline Zanthoxylum fagara (L.) Sarg. & $\mathrm{t}$ & Marchesi et al. s.n.(MVFA 28054) \\
\hline Z. rhoifolium Lam. & $\mathrm{t}$ & Marchesi 846 (MVFA) \\
\hline Zanthoxylum sp. & $\mathrm{t}$ & Brussa et al. s.n.(MVJB 23665) \\
\hline \multicolumn{3}{|l|}{ SALICACEAE } \\
\hline Azara uruguayensis (Speg.) Sleum. & $s / t$ & Del Puerto s.n.(MVFA 2253) \\
\hline Banara tomentosa Clos & $\mathrm{t}$ & Grela \& Brussa 1410 (MVJB 22299) \\
\hline B. umbraticola Arechav. & $\mathrm{t}$ & Brussa \& Grela s.n.(MVJB 25082) \\
\hline Casearia decandra Jacq. & $\mathrm{t}$ & Brussa \& Grela s.n.(MVFA 29150) \\
\hline C. sylvestris Sw. & $\mathrm{t}$ & Brescia et al. s.n.(MVFA 16291) \\
\hline Salix humboldtiana Willd. & $\mathrm{t}$ & Rosengurtt et al. PE-5552 (MVFA) \\
\hline Xylosma pseudosalzmannii Sleumer & $\mathrm{t}$ & Brussa \& Grela s.n.(MVFA 32662) \\
\hline X. schroederi Sleumer ex Herter & $s / t$ & Brussa s.n.(MVJB 24338) \\
\hline X. tweediana (Clos) Eichl. & $s / t$ & Rabaiotti \& Grela s.n.(MVFA 24302) \\
\hline X. venosa N.E.Br. & $\mathrm{t}$ & Brussa \& Grela s.n.(MVJB 24864) \\
\hline \multicolumn{3}{|l|}{ SANTALACEAE } \\
\hline Acanthosyris spinescens (Mart. \& Eichl.) Griseb. & $\mathrm{t}$ & Marchesi 1294 (MVFA) \\
\hline Jodina rhombifolia (Hook. \& Arn.) Reissek & $\mathrm{t}$ & Rosengurtt et al. PE-5545 (MVFA) \\
\hline \multicolumn{3}{|l|}{ SAPINDACEAE } \\
\hline $\begin{array}{l}\text { Allophylus edulis (A. St.-Hil., A. Juss. \& Cambess.) Hieron. ex } \\
\text { Niederl. }\end{array}$ & $\mathrm{t}$ & Rosengurtt \& Gallinal 5686 (MVFA) \\
\hline A. guaraniticus (A. St.-Hil.) Radlk. & $\mathrm{s} / \mathrm{t}$ & Brussa et al. s.n.(MVJB 21525) \\
\hline Cupania vernalis Cambess. & $s / t$ & Legrand $2367(M V M)$ \\
\hline Dodonaea viscosa (L.)Jacq. & $\mathrm{s} / \mathrm{t}$ & Rosengurtt B-45375 (MVFA) \\
\hline Matayba eleagnoides Radlk. & $\mathrm{t}$ & Brescia et al. s.n.(MVFA 19057) \\
\hline \multicolumn{3}{|l|}{ SAPOTACEAE } \\
\hline Chrysophyllum gonocarpum (Mart. \& Eichl.) Engl. & $\mathrm{t}$ & Rosengurtt B-6915 (MVFA) \\
\hline C. marginatum (Hook. \& Arn.) Radlk. & $\mathrm{t}$ & Brescia \& Grun s.n.(MVFA 22523) \\
\hline Pouteria gardneriana (A.DC.) Radlk. & $\mathrm{t}$ & Brussa et al. s.n.(MVJB 21587) \\
\hline P. salicifolia (Spreng.) Radlk. & $\mathrm{t}$ & Rosengurtt et al. PE-5609 (MVFA) \\
\hline Sideroxylon obtusifolium (Roemer \& Schult.) T.D.Penn. & $s / t$ & Figueredo s.n.(MVJB 20274) \\
\hline \multicolumn{3}{|l|}{ SCROPHULARIACEAE } \\
\hline $\begin{array}{l}\text { Buddleja elegans Cham. \& Schltdl. ssp. angustata (Benth.) } \\
\text { E.M.Norman }\end{array}$ & s & Brussa \& Grela s.n.(MVJB 24996) \\
\hline B. grandiflora Cham. \& Schltdl. & s & Rosengurtt B-4999 (MVFA) \\
\hline B. stachyoides Cham. \& Schltdl. & s & Rosengurtt B-4108 (MVFA) \\
\hline B. thyrsoides Lam. & s & Berro 3797 (MVFA) \\
\hline \multicolumn{3}{|l|}{ SIMAROUBACEAE } \\
\hline Castela tweediei Planch. & $s / t$ & Rosengurtt B-2246 (MVFA) \\
\hline \multicolumn{3}{|l|}{ SOLANACEAE } \\
\hline Cestrum euanthes Schltdl. & s & Brussa et al. s.n.(MVJB 27150) \\
\hline C. parqui L'Her. & s & Del Puerto \& Rosell s.n.(MVFA 892) \\
\hline C. strigilatum Ruiz \& Pav. & s & Del Puerto \& Marchesi s.n.(MVFA 3643) \\
\hline Grabowskia duplicata Arn. & s & Marchesi \& Armand-Ugón s.n.(MVFA 21159) \\
\hline Lycium cestroides Schltdl. & s & Rosengurtt B-3647 (MVFA) \\
\hline
\end{tabular}


Table 1. Continuation.

\begin{tabular}{|c|c|c|}
\hline Family / Species & Life form & Reference specimen \\
\hline L. ciliatum Schltdl. & s & Marchesi s.n.(MVFA 21867) \\
\hline L. vimineum Miers & s & Berro 1438 (MVFA) \\
\hline Solanum bonariense $\mathrm{L}$. & s & Arrillaga et al. 1976 (MVFA) \\
\hline S. glaucophyllum Desf. & s & Arrilla \& Izaguirre 2648 (MVFA) \\
\hline S. granuloso-leprosum Dunal & $s / t$ & Rosengurtt B-1984 (MVFA) \\
\hline S. mauritianum Scop. & $s / t$ & Arrillaga 815 (MVFA) \\
\hline S. sanctae-catharinae Dunal & $\mathrm{t}$ & Arrillaga et al. 1859 (MVFA) \\
\hline Vassobia breviflora (Sendtn.) A.T.Hunziker & $s / t$ & Berro 3338 (MVFA) \\
\hline \multicolumn{3}{|l|}{ STYRACACEAE } \\
\hline Styrax leprosus Hook. \& Arn. & $s / t$ & Rosengurtt B-5319 (MVFA) \\
\hline \multicolumn{3}{|l|}{ SYMPLOCACEAE } \\
\hline Symplocos uniflora (Pohl) Benth. & $\mathrm{t}$ & Marchesi \& Baycé s.n.(MVFA 19986) \\
\hline \multicolumn{3}{|l|}{ THYMELAEACEAE } \\
\hline Daphnopsis racemosa Griseb. & s & Rosengurtt \& Gallinal 5897 (MVFA) \\
\hline \multicolumn{3}{|l|}{ VERBENACEAE } \\
\hline Aloysia chamaedryfolia Cham. & s & Masciadri s.n.(MVJB 27227) \\
\hline A. gratissima (Gillies \& Hook.) Tronc. & s & Rosengurtt A-409 (MVFA) \\
\hline A. pulchra (Briq.) Mold. & s & Izaguirre et al. s.n.(MVFA 21398) \\
\hline Citharexylum montevidense (Spreng.) Mold. & $\mathrm{t}$ & Legrand $687(M V M)$ \\
\hline Duranta erecta $\mathrm{L}$. & s & Schroeder (H. Osten 15511) (MVM) \\
\hline Lantana cf. camara L. & s & Rosengurtt B-2915 (MVFA) \\
\hline L. megapotamica (Spreng.) Tronc. & s & Lombardo 6420 (MVJB 9923) \\
\hline Lippia alba (Mill.) N.E.Br. & s & Marchesi 1057 (MVFA) \\
\hline \multicolumn{3}{|l|}{ ZYGOPHYLLACEAE } \\
\hline Porlieria microphylla (Baill.) Desc. et al. & s & Gómez s.n.(MVM 1145) \\
\hline
\end{tabular}

per quadrant in the Eastern A and the Eastern B regions, respectively (Fig. 2). The geographic distribution of records within each region is quite heterogeneous (Fig. 3), with some areas showing high concentration of records and others with a few to no records at all. The proportion of sufficiently sampled quadrants ascend to $7.5 \%$ in the Western region, $7.7 \%$ in the Central region, $13.6 \%$ in the Eastern A region and $9.1 \%$ in the Eastern B region. Quadrants without records (i.e., information gaps) represent 10\%, 29.4\%, 9.1\% and $18.2 \%$ of each of these regions, respectively, and $7 \%$ of the whole country.

\section{Similarity between tree species of Uruguay and neighboring regions}

Uruguayan tree species richness was similar to that registered for the Entre Ríos Province, which was higher than that of the Buenos Aires Province but much lower than that of Rio Grande do Sul State (Table 2). Indeed, Rio Grande do Sul has the highest values for families, genera and species richness of the four compared regions (Table 2). Tree richness per surface unit indicates values for Uruguay that are four times higher than that of Buenos Aires, and about a half of that of Rio Grande do Sul (Table 2). The similarity analysis among regions shows greater similitude of Uruguayan tree flora with Entre Ríos than any of the other regions and the lowest similarity with Rio Grande do Sul (Table 3).

\section{Discussion}

This work generated precise definitions of trees and shrubs appropriate for the Uruguayan context that, despite being arbitrary, attempt to avoid the ambiguity and possible confusion when compiling species lists. The difference between the species list reported here and the list generated by Lombardo (1964) is easily explained by new records of species for the Uruguayan flora reported more or less continuously during the last 46 years (Table 4 ). With regards to Brussa \& Grela (2007), the number of species is similar to that presented here, but there is a considerable difference (24\%) in species composition. This difference is mainly due to the lack of explicit criteria for inclusion of species in the aforementioned publication, resulting in the exclusion of 

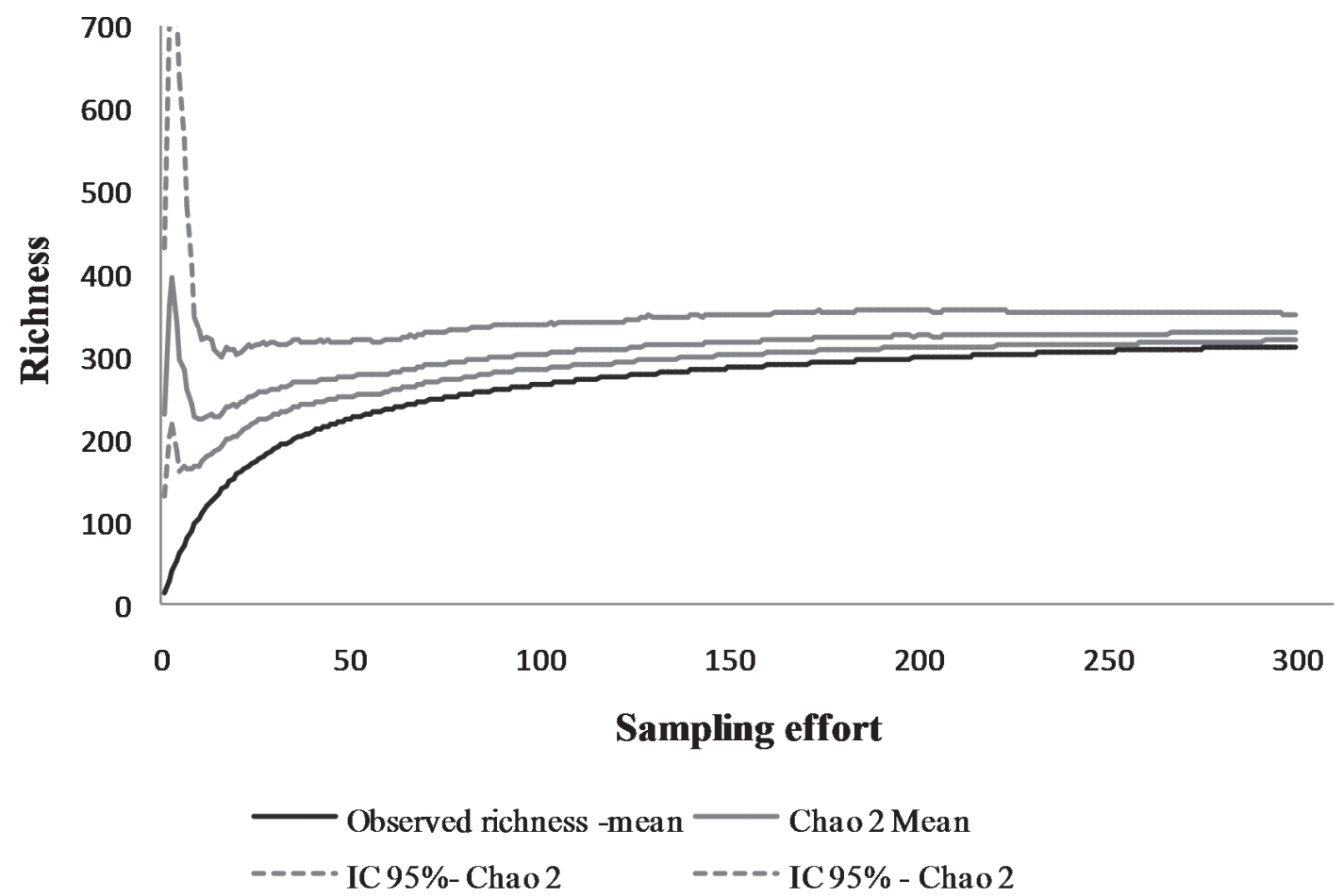

Figure 1. Cumulative richness of tree and shrub species in Uruguay based on the distribution of records. The estimated richness was calculated with Chao 2 index. IC = confidence interval of $95 \%$.
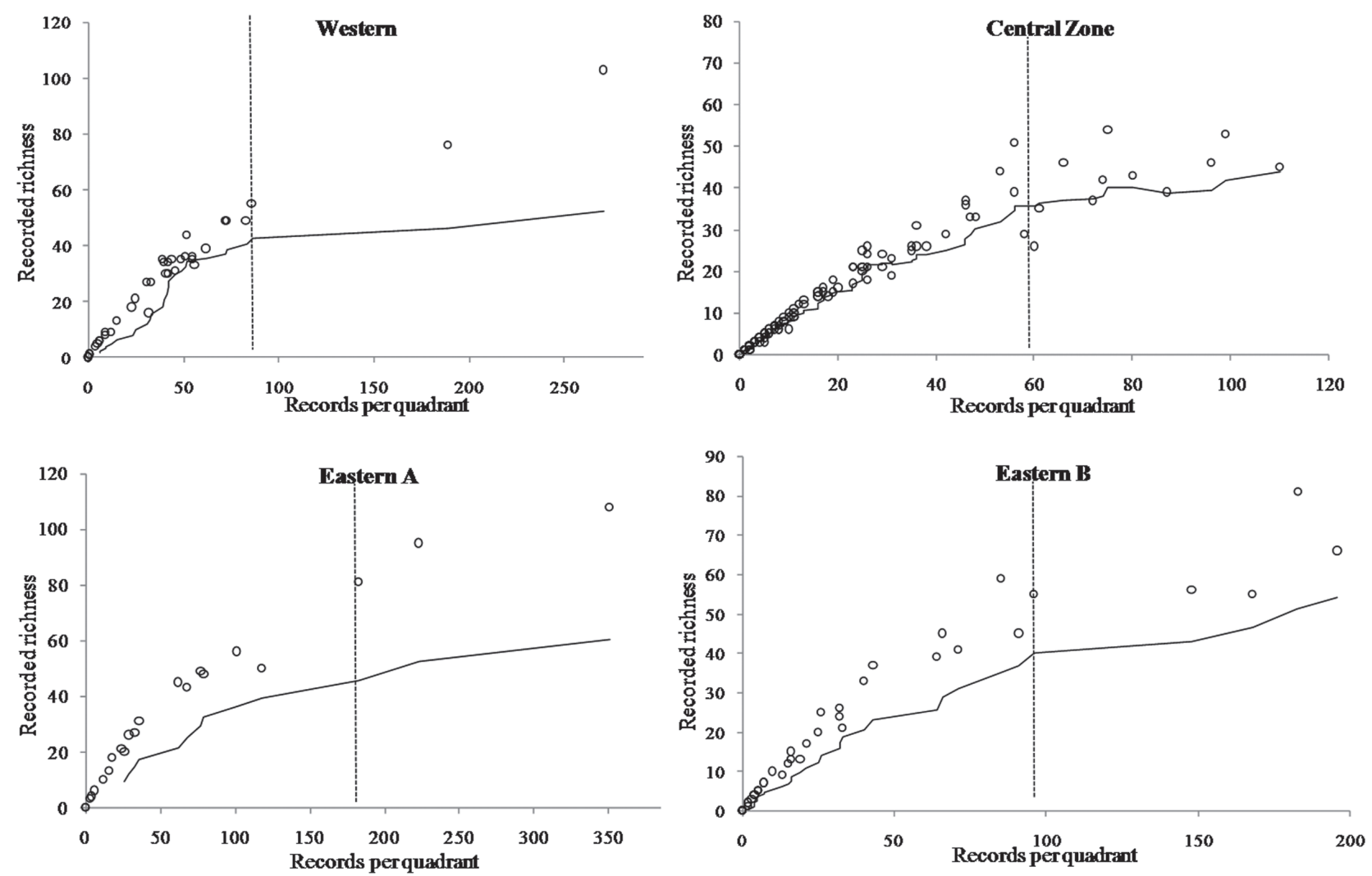

Figure 2. Relation between observed richness per quadrant and the sampling effort (number of records) for each region. The solid curve was smoothing by moving average (lag: 10). The dotted line shows the value where the sampling effort starts the saturation. 

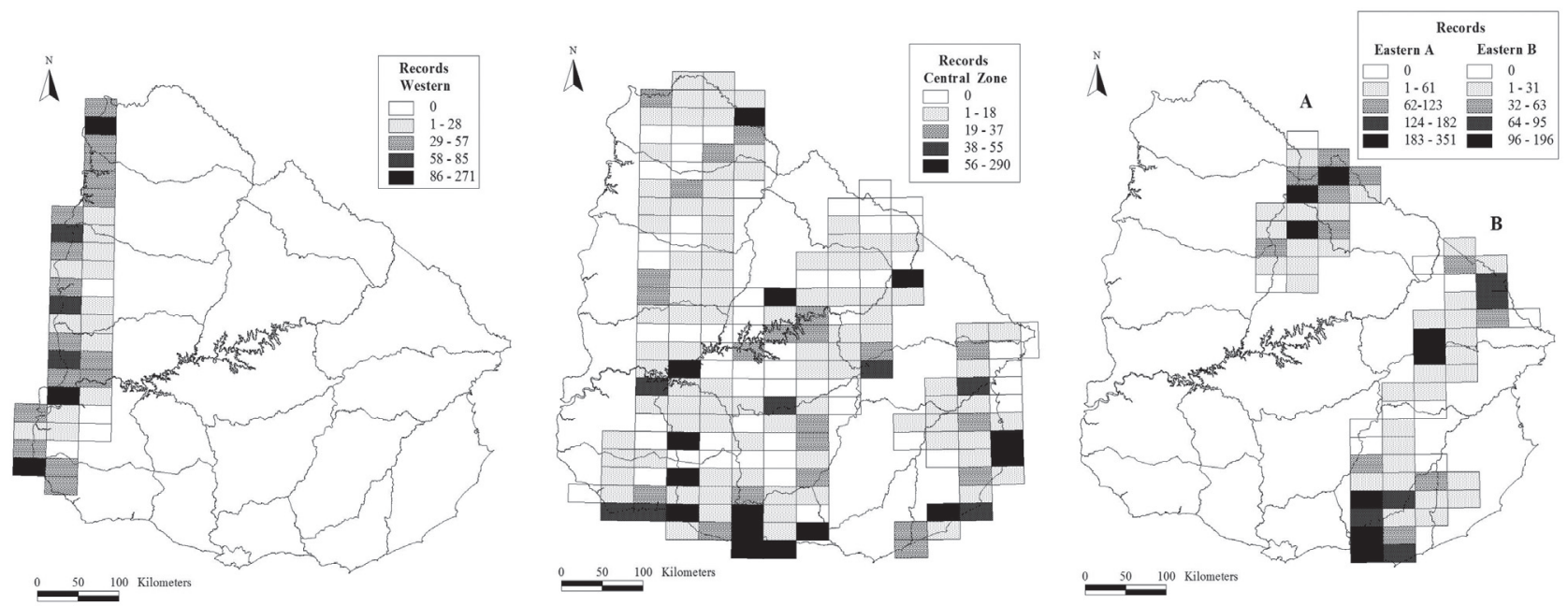

Figure 3. Geographic distribution of sampling effort by quadrant in Uruguay for each region.

Table 2. Richness of trees at the level of families, genus and species and tree density in Uruguay and neighboring regions. Data of neighboring regions from Sobral et al. 2006; Zuloaga et al. (2008).

\begin{tabular}{lcccc}
\hline & Families & Genera & Species & $\begin{array}{c}\text { Species } \\
/ 1000 \mathrm{~km}^{2}\end{array}$ \\
\hline Buenos Aires & 30 & 50 & 62 & 0,20 \\
Entre Ríos & 35 & 84 & 106 & 1,34 \\
Uruguay & 46 & 105 & 151 & 0,86 \\
$\begin{array}{l}\text { Rio Grande } \\
\text { do Sul }\end{array}$ & 79 & 238 & 510 & 1,81 \\
\hline
\end{tabular}

Table 3. Similarity matrix of tree species among Uruguay (UR), Rio Grande do Sul (RS), Entre Ríos (ER) and Buenos Aires (BS), Jaccard Index.

\begin{tabular}{ccccc}
\hline & UR & RS & ER & BS \\
\hline UR & 1,00 & 0,27 & 0,53 & 0,34 \\
RS & & 1,00 & 0,16 & 0,09 \\
ER & & & 1,00 & 0,50 \\
BS & & & & 1,00 \\
\hline
\end{tabular}

Table 4. Number of tree and shrub species and families, proposed by different authors and by the present work.

\begin{tabular}{lcc}
\hline Author & Species & Families \\
\hline Lombardo (1964) & 236 & 52 \\
Alonso y Bassagoda (2002) & 254 & 54 \\
Brussa \& Grela (2007) & 302 & 55 \\
Present work & 313 & 57 \\
\hline
\end{tabular}

species when our definitions are applied (Table 4). In addition, the work of Brussa \& Grela (2007) emphasizes species from Tacuarembó and Rivera, thus the current list includes several species elsewhere in the country not present in that work. We also excluded some species present in Brussa \&
Grela (2007) because their presence in Uruguay has not been confirmed or for lack of taxonomic updates or knowing the exact location of specimens.

Alternatively, the information for some taxonomic groups (e.g., Baccharis L., Croton L., Opuntia Mill.) still remains incomplete due to the high taxonomic complexity and elevated diversity of Baccharis and Croton in Uruguay, as well as the lack of specimens of Opuntia. So, new species may be added to the list of trees and shrubs present in the country. Despite the fact that the number of taxonomic works focused on woody species has increased in the last few years, there are rather few, thus stressing the need for further studies that address untreated families and genera.

From the standpoint of inventory completeness, our results suggest that the accumulated knowledge of trees and shrubs of Uruguay is fairly complete, with only $11 \%$ of the potential number of woody species yet to be registered.

The floristic regional completeness within Uruguay allows a more accurate approximation of the sampling effort needed in areas of the country with different environmental characteristics. In all regions there is more or less heterogeneity of the sampling effort, with areas with scarce or no information. The Eastern A region has the highest degree of completeness, while the Central region has the highest proportion of gaps. This situation results from a lack of planned and systematic sampling, a common problem in many areas (Spichiger et al. 2004). Instead, ease of access and landscape attractiveness has generated the high concentration of sampling in some areas while neglecting others. The low degree of completeness in the Central region is echoed in other biological groups (Brazeiro et al. 2008; Canavero et al. 2010). Apart from the Central region the analysis presented here allowed us to identify other areas with scarce completeness, whose study should be marked as a priority in future studies and surveys. 
The comparison of Uruguayan tree species richness with neighboring regions offers further support for the idea that there are differences between Uruguay and the Province of Buenos Aires flora (Chebataroff 1942; 1960; Cabrera \& Willink 1973; Del Puerto 1987; Alonso \& Bassagoda 2002; Grela 2004), which is consistent with the demarcation of a Uruguayan District inside the Pampean Province as proposed by Cabrera \& Willink (1973).

The results of the similarity analysis and the species composition evaluation emphasize the transitional nature of the Uruguayan flora between the Pampas grasslands, and the Chaco and Paranaense forests. The influence of the Paranaense Province is manifested by the presence of characteristic species, such as Nectandra megapotamica (Spreng.) Mez, Peltophorum dubium (Spreng.) Taub., Parapiptadenia rigida (Benth.) Brenan, Handroanthus heptaphyllus (Vell.) Mattos, Syagrus romanzoffiana (Cham.) Glassm., Ilex paraguariensis A.St.-Hil. and Ficus cestrifolia Schott (Cabrera \& Willink 1973; Fiaschi \& Pirani 2009). Moreover, several species indentified as dominant or characteristic of the Paranaense Province in southern Brazil and northeastern Argentina (Benvenuti-Ferreira \& Cohelo 2009; Grings \& Brack 2009; Klauberg et al. 2010; Ruschel et al. 2005; 2009) are also present in Uruguay (e.g., Actinostemon concolor (Spreng.) Müll. Arg., Campomanesia xanthocarpa (Mart.) O.Berg, Casearia decandra Jacq., Chrysophyllum gonocarpum (Mart. \& Eichl.) Engl., Cupania vernalis Cambess., Dicksonia sellowiana Hook., Luehea divaricata Mart., Matayba elaeagnoides Radlk., Myrcianthes gigantea (D.Legrand) D.Legrand, Ocotea pulchella Mart., Ocotea puberula (Rich.) Nees, Ruprechtia laxiflora Meisn., Zanthoxylum rhoifolium Lam.). Furthermore, affinity with the Chaco and Espinal floras is evident from the presence of species in Uruguay which are also dominant in these Provinces (e.g., Vachellia caven (Molina) Seigler \& Ebinger, Senegalia praecox (Griseb.) Seigler \& Ebinger, Aspidosperma quebracho-blanco Mart. \& Zucc., Butia yatay (Mart.) Becc., Celtis ehrenbergiana (Klotzsch) Liebm., Geoffroea decorticans (Gillies ex Hook. \& Arn.) Burkart, Jodina rhombifolia (Hook. \& Arn.) Reissek, Maytenus vitis-idaea Griseb., Prosopis affinis Spreng., P. nigra (Griseb.) Hieron., Schinus longifolius (Lindl.) Speg., Scutia buxifolia Reissek, Trithrinax campestris (Burmeist.) Drude \& Griseb. (Cabrera \& Willink 1973; Kopta 1999; Marino \& Pensiero 2003).

The transitional nature of the Uruguayan vegetation and flora explains the relatively high tree and shrub species richness, especially when the small continental land area of the country and particularly the scarce native forest surface are taken into account. The Uruguayan territory represents the southernmost and southeastern boundaries for the distribution of many species, with some showing restricted distribution within the country (Alonso \& Bassagoda 2002; Brussa \& Grela 2007).

In conclusion, despite the fact that Uruguay lies within a region dominated by grasslands, the country has a surprisingly high richness of tree and shrub species and its woody flora shows considerable differences with those of neighboring regions. The difference with the typical Pampean flora analyzed is remarkable, represented by Buenos Aires. Detailed future studies of the woody flora and vegetation will allow re-evaluating the proposed phytogeographic schemes and shedding further light on the plant distribution patterns for the region.

\section{Acknowledgements}

We thank the herbarium curators for making the collections available. We are also grateful to Carlos Brussa and Liliana Delfino for their critical reviews of the early version of the manuscript, and to Mauricio Bonifacino and Christine Lucas for their valuable comments and idiomatic revision. We also appreciate the critical reviews of three anonymous referees. This study was financially supported by the Comisión Sectorial de Investigación Cientifica (CSIC) of the Universidad de la República, Uruguay (Project N 805-102).

\section{References}

Alonso, E. \& Bassagoda, M.J. 1999. Los bosques y los matorrales psamófilos en el litoral platense y atlántico del Uruguay. Comunicaciones Botánicas del Museo de Historia Natural de Montevideo VI (113).

Alonso, E. \& Bassagoda, M. J. 2002. Aspectos fitogeográficos y diversidad biológica de las formaciones boscosas del Uruguay. Ciência e ambiente 24: 35-48.

APG III. 2009. An update of the Angiosperm Phylogeny Group classification for the orders and families of flowering plants: APG III. Botanical Journal of the Linnean Society 161: 105-121.

Arechavaleta, J. 1898-1911. Flora Uruguaya. Enumeración y descripción breve de las plantas conocidas hasta hoy y de algunas nuevas que nacen espontáneamente y viven en la República Uruguaya. Anales del Museo Nacional de Montevideo III-VII.

Arechavaleta, J. 1906. Reseña de los botánicos que herborizaron en esta región al oriente del Uruguay. En: Flora Uruguaya. Tomo II. Anales del Museo Nacional de Montevideo 5: XXXIV-XLIII.

Arechavaleta, J. 1908a. Complemento a la reseña histórica del tomo II página XXXIX. In: Flora Uruguaya Tomo III. Anales del Museo Nacional de Montevideo 6: 1-7.

Arechavaleta, J. 1908b. Naturalistas en el Uruguay. Revista Histórica de la Universidad I: 478-506; 828-842. Montevideo.

Arrillaga, B.; Ziliani, G. \& Ren, J. 1973. Anacardiáceas de Uruguay. Boletín de Investigaciones 126. Montevideo, UDELAR-Facultad de Agronomía.

Benvenuti-Ferreira, G. \& Coelho, G.C. 2009. Floristics and structure of the tree component in a Seasonal Forest remnant, Chiapetta, Rio Grande do Sul State, Brazil. Revista Brasileira de Biociências 7(4): 344-353.

Brazeiro, A.; Achkar, M.; Canavero, A.; Fagúndez, C.; González, E.; Grela, I.; Lezama, F.; Maneyro, R.; Bartesaghi, L.; Camargo, A.; Carreira, S.; Costa, B.; Núñez, D.; da Rosa, I. \& Toranza, C. 2008. Prioridades geográficas para la conservación de la biodiversidad terrestre de Uruguay. Resumen ejecutivo. Proyecto PDT 32-26. Available in: http:// www.universidadur.edu.uy/retema/archivos/PrioridadesGeograficasConservacion_2008.pdf

Brussa, C. \& Grela, I. 2007. Flora arbórea del Uruguay. Con énfasis en las especies de Rivera y Tacuarembó. Cofusa. Montevideo.

Cabrera, A.L. \& Willink, A. 1973. Biogeografía de América Latina. Washington D.C., Secretaría General de la Organización de Estados Americanos. Programa Regional de Desarrollo Científico y Tecnológico, Departamento de Asuntos Científicos. Serie Biología 13. 
Canavero, A.; Brazeiro, A.; Camargo, A.; Da Rosa, I.; Maneyro, R. \& Núñez, D. 2010. Amphibian diversity of Uruguay: background knowledge, inventory completeness and sampling coverage. Boletín de la Sociedad de Zoología de Uruguay 19:1-19.

Chebataroff, J. 1942. La vegetación del Uruguay y sus relaciones fitogeográficas con la del resto de la América del Sur. Revista Geográfica del Instituto Panamericano de Geografía e Historia, México: 50-90.

Chebataroff, J. 1960. Algunos aspectos evolutivos de la vegetación de la Provincia Fitogeográfica Uruguayense. Revista Nacional 201: 1-18.

Colwell, R. 2006. EstimateS: Statistical estimation of species richness and shared species from samples. Version 8.0. Department of Ecology and Evolutionary Biology, University of Connecticut Storrs. Connecticut, EEUU.

Crisci, J. 2006. Espejos de nuestra época: biodiversidad, sistemática y educación. Gayana Botánica 63(1): 106-114.

Del Puerto, O. 1969. Hierbas del Uruguay. Nuestra Tierra (19).

Del Puerto, O. 1987. Vegetación del Uruguay. Montevideo, Facultad de Agronomía.

Fiaschi P. \& Pirani J.R. 2009. Review of plant biogeographic studies in Brazil. Journal of Systematics and Evolution 47 (5): 477-496.

Font Quer, P.1957. Diccionario de Botánica. España, Ediciones Península.

Funk, V. 2006. Floras: a model for biodiversity studies or a thing of the past? Taxon 55(3): 581-588.

Gibert, E. 1873. Enumeratio plantarum sponte nascientium agro montevidensis. Asociación Rural del Uruguay, Montevideo.

Grela, I. 2004. Geografía florística de especies arbóreas de Uruguay: propuesta para la delimitación de dendrofloras. Tesis M.Sc. PEDECIBAUniversidad de la República. Montevideo, Uruguay.

Grings, M.; Brack, G. 2009. Árvores na vegetação nativa de Nova Petrópolis, Rio Grande do Sul. Iheringia, Série Botânica 64 (1): 5-22.

Hammer, O.; Harper, D. A. T. \& Ryan, P. D. 2001. PAST: Paleontological Statistics software package for education and data analysis. Palaeontologia Electronica 4(1)

Herter, G. 1930. Estudios Botánicos en la Región Uruguaya IV. Florula uuruguayensis. Plantae Vasculares. Edición Nacional Centenario, Montevideo.

Herter, G. 1943-1957. Flora Ilustrada del Uruguay. Vol. 2. Montevideo, Berlín, Cracovia.

Holmgren, PK, Holmgren NH. 1998. [continuously updated]. Index Herbariorum: A global directory of public herbaria and associated staff. New York Botanical Garden's Virtual Herbarium. http:// sweetgum.nybg.org/ih/

Izaguirre, P. \& Beyhaut, R. 1998. Las Leguminosas en Uruguay y regiones vecinas. Parte I: Papilionoideae. Montevideo, Hemisferio Sur.

Izaguirre, P. \& Beyhaut, R. 2003. Las Leguminosas en Uruguay y regiones vecinas. Parte II: Caesalpinoideae. Parte III: Mimosoideae. Montevideo, Hemisferio Sur.

Klauberg, C.; Festa Paludo, G.; Lopes da Costa Bortoluzzi, R. \& Mantovani A. 2010. Florística e estrutura de um fragmento de Floresta Ombrófila Mista no Planalto Catarinense. Biotemas 23(1): 35-47.

Kopta, F. 1999. Disminución de la flora autóctona. Pp. 203. In: Problemática ambiental con especial referencia a la Provincia de Córdoba. Córdoba, Fundación Ambiente, Cultura y Desarrollo - ACUDE. UNESCO y Embajada Real de los Países Bajos.

Larrañaga, D.A. 1922. Escritos. Montevideo, Instituto Histórico y Geográfico del Uruguay, Tomo I. Imprenta Nacional.

Larrañaga, D.A. 1923. Escritos. Montevideo, Instituto Histórico y Geográfico del Uruguay, Tomo II. Imprenta Nacional.

Larrañaga, D.A. 1927. Atlas 1, Botánica. Montevideo, Instituto Histórico y Geográfico del Uruguay. Imprenta Nacional.

Legrand, D. 1936. Las Mirtáceas del Uruguay I. Anales del Museo de Historia Natural de Montevideo Serie 2, 4(11):1-70.
Legrand, D. 1943. Las Mirtáceas del Uruguay, II. Comunicaciones botánicas del Museo de Historia Natural de Montevideo 1(7): 1-11.

Legrand, D. 1968. Las Mirtáceas de Uruguay, III. Boletín de la Facultad de Agronomía 101: 1-80.

Lista de Espécies da Flora do Brasil 2011 in http://floradobrasil.jbrj.gov. br/2011 (visited 1/2012).

Lombardo, A. 1946. Flora arbórea y arborescente del Uruguay. Montevideo, Laboratorios Galien.

Lombardo, A. 1964. Flora arbórea y arborescente del Uruguay. 2 ed. Montevideo, Concejo Departamental de Montevideo, Dirección de Paseos Públicos.

Lombardo, A. 1982-1984. Flora Montevidensis I-III. Intendencia Municipal de Montevideo, Servicio de Publicaciones y prensa.

Marchesi, E. 1983. Catálogo preliminar de la Flora Uruguaya. Lauraceae. Revista de la Asociación de Ingenieros Agrónomos 1(1): 55-57.

Marino, G.D.; Pensiero, J.F. 2003. Heterogeneidad florística y estructural de los bosques de Schinopsis. Darwiniana 41(1-4): 17-28.

MGAP. 2011. http://www.mgap.gub.uy/Forestal/DGF.htm (visited 1/2011).

Mori, S. A. 1992. Neotropical Floristics and Inventory: Who will do the work? Brittonia 44(3): 372-375.

Morrone, J. J. 2001. Biogeografía de América Latina y el Caribe. Vol. 3. CYTED- ORCYT/ UNESCO - SEA- Corporación Iberoamericana. Manuales y Tesis.

Muñoz, J.; Ross, P. \& Cracco, P. 1993. Flora indígena del Uruguay: árboles y arbustos ornamentales. Montevideo. Hemisferio Sur.

Muñoz, J.; Ross, P. \& Cracco, P. 2005. Flora indígena del Uruguay: árboles y arbustos ornamentales. 2 ed. Montevideo, Hemisferio Sur.

Palmer, M.W.; Wade, G.L. \& Neal, P. 1995. Standards for the Writing of Floras BioScience 45 (5): 339-345.

Prance, G.T.; Beentje, H.; Dransfield, J. \& Source, R.J. 2000. The Tropical Flora Remains Undercollected. Annals of the Missouri Botanical Garden 87(1): 67-71.

Ricker, M. \& Hernández, H.M. 2010. Tree and tree-like species of Mexico: gymnosperms, monocotyledons, and tree Ferns. Revista Mexicana de Biodiversidad 81: 27- 38 .

Ruschel, A.; Guerra, M.; Moerschbacher, B. \& Nodari, R. 2005. Valuation and characterization of the timber species in remnants of the Alto Uruguay River ecosystem, southern Brazil. Forest Ecology and Management 217: 103-116.

Ruschel, A.; Guerra, M. \& Nodari, R. 2009. Estrutura e Composição Florística de Dois Fragmentos da Floresta Estacional Decidual do Alto-Uruguai, SC. Ciência Florestal 19(2): 225-236.

Schnak J.A. \& López H.L. 2003. Biodiversidad, iniciativa global y elaboración de Inventarios Sistemáticos. ProBiota, Serie Técnica y Dicáctica 3: 1-14.

Smith, N.; Mori, S.; Henderson, A.; Steveson, D. \& Heald, S. 2004. Flowering plants of the Neotropics. The New York Botanical Garden. Princeton, Princeton University Press.

Sobral, M.; Jarenkow, J.A.; Brack, P.; Irgang, B.; Larocca, J. \& Schütz Rodrigues, R. 2006. Flora arbórea y arborescente de Rio Grande do Sul. São Carlos, RiMa, Novo Ambiente.

Spichiger, R.; Calenge, C. \& Bise, B. 2004. Geographical zonation in the Neotropics of tree species characteristic of the Paraguay-Paraná Basin. Journal of Biogeography 31: 1489-1501.

Tropicos.org. 2011. http://www.tropicos.org/ (visited 1/2011).

Zuloaga, F.O.; Morrone, O.; Belgrano, M.J.; Marticorena, C. \& Marchesi, E. (Eds.) 2008. Catálogo de las Plantas Vasculares del Cono Sur (Argentina, Sur de Brasil, Chile, Paraguay y Uruguay). Monographs in Systematic Botany from the Missouri Botanical Garden 107(1): i-xcvi, 1--983; 107(2): i--xx, 985--2286; 107(3): i--xxi, 2287--3348. 